\title{
Decreased brain-expressed X-linked 4 (BEX4) expression promotes growth of oral squamous cell carcinoma
}

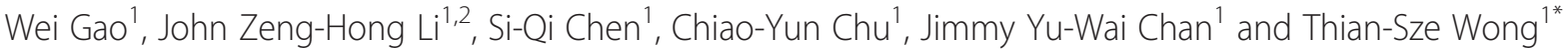

\begin{abstract}
Background: Brain-expressed X-linked (BEX) 4 is a member of BEX family. The functional role of BEX4 in oral squamous cell carcinoma (OSCC) remains unknown.

Methods: Expression level of BEX family members (BEX1-5) in OSCC tissues and the paired normal epithelial were examined. Functions of epigenetic changes (DNA methylation and histone modifications) on BEX4 suppression in OSCC were examined by zebularine and trichostatin A (TSA) treatment on OSCC cell lines. Lentivector containing full-length BEX4 was used to generate OSCC cell lines with stable BEX4 expression. Effects of BEX4 expression on OSCC proliferation were monitored with XCELLigence RTCA real-time cell analyzer. BEX4-overexpressing CAL27 was implanted into nude mice to evaluate the effects on tumor growth in vivo. The signaling pathways regulated by BEX4 in OSCC was explored using human whole-transcript expression microarray.

Results: Among the 5 BEX family members, BEX1 and BEX4 showed significant down-regulation in OSCC $(P<0.001)$. $B E X 3$, in comparison, was overexpressed in the primary tumor. BEX4 expression in OSCC cell lines was re-activated after zebularine and TSA treatment. High BEX4 expression could suppress proliferation of OSCC in vitro. Subcutaneous tumor volume of BEX4-overexpressing CAL27 was remarkably reduced in nude mice. Microarray experiment showed that S100A family members (S100A7, S100A7A, S100A8, S100A9 \& S100A12) might be the downstream targets of BEX4 in OSCC.
\end{abstract}

Conclusions: BEX4 functions as tumor suppressor by inhibiting proliferation and growth of oral cancer. Decreased BEX4 contributes to the increased proliferative propensity of OSCC.

Keywords: Oral cancer, Squamous cell carcinoma, Brain-expressed X-linked family, BEX4, Tumor growth, S100A family

\section{Background}

Epithelial cancer derived from the oral mucosa is a common head and neck cancer [1]. Histologically, oral squamous cell carcinoma (OSCC) is the major subtype contributing to over $90 \%$ of the cases [2]. Despite the advances in cancer detection techniques and treatment methods in the past decade, the incidence of OSCC remains high and the 5-year survival rate of OSCC patients remains unsatisfactory [3]. OSCC is an aggressive head and neck cancer due to the rapid proliferative capability and high invasive nature [4]. The survival rate of

\footnotetext{
* Correspondence: thainsze@gmail.com

'Department of Surgery, The University of Hong Kong, Hong Kong SAR, China

Full list of author information is available at the end of the article
}

OSCC patients showed close association with the tumor size [5]. It has been shown that tumor size is an independent predictive factor for survival of OSCC patients [6]. Therefore, a better understanding on the underlying mechanisms involve in OSCC growth is important to improve the prognosis of OSCC patients.

Silencing key tumor suppressor genes by epigenetic mechanisms are essential for the carcinogenic development of OSCC. Identifying key methylated genes with functional implications in the development of OSCC would be useful in diagnosis and as therapeutic targets [7]. High-throughput parallel screening using expression and methylation microarrays had shown that brainexpressed X-linked (BEX) members were susceptible to 
epigenetic inactivation in OSCC [8]. Human BEX members (BEX1, BEX2, BEX3, BEX4, BEX5) are all located on chromosome Xq22. They are highly conserved genes among different species (including chimp, mouse, rat, dog and human) implying their importance in cellular functions [9]. At present, little is known about the functional implications of BEX family members in the progression of human malignancies. It has been reported that BEX family members could regulate cell cycle and apoptotic signaling $[10,11]$.

BEX family members displayed distinct tissue distribution and have varying roles dependent on different cellular context. Thus, in the present study, we first explored the expression patterns of the human BEX family members in OSCC. Given that BEX4 expression was remarkably reduced in our cohort, we hypothesized that BEX4 could possibly play a tumor-suppressing role in OSCC. Further, whether DNA methylation or chromatin remodeling would be a possible cause of BEX4 suppression in OSCC will also be investigated. The functional role of BEX4 on tumor growth was examined using OSCC cell lines and xenograft models. As there was no information about the functional role of BEX4 in human malignancies, global gene expression change of BEX4-overexpressing OSCC cell line will be explored using microarray.

\section{Methods}

\section{Cell cultures}

Human tongue squamous cell carcinoma cell line CAL27 was obtained from American Type Culture Collection. Buccal squamous cell carcinoma cell line YD-38 was obtained from Korean cell line bank. Both cell lines were maintained in RPMI 1640 medium supplemented with $10 \%$ fetal bovine serum, $100 \mathrm{U} / \mathrm{ml}$ penicillin and $100 \mu \mathrm{g} /$ $\mathrm{ml}$ streptomycin. Drug treatment started after cell seeding for $24 \mathrm{~h}$. For zebularine treatment, the cells were harvested after $72 \mathrm{~h}$. For trichostatin A (TSA) treatment, the cells were harvested after $24 \mathrm{~h}$.

\section{Clinical samples}

Sixty-five OSCC and the paired normal tissues were examined. Tissue were obtained from patients underwent surgery in Division of Head and Neck, Queen Mary Hospital, Hong Kong. All the cancer tissues were squamous cell carcinoma. Consent of tissue donation for research purpose was obtained before sample collection. The study was approved by the ethnic review board of our institute (registered number: UW12-123).

\section{RNA extraction and real-time quantitative polymerase chain reaction (qPCR)}

Total RNA was extracted using Trizol reagent (Invitrogen). First-strand cDNA synthesis was performed using High Capacity cDNA Reverse Transciption Kit (ABI).
Transcript level was evaluated by qPCR using FastStart Universal Probe Master (Roche Applied Science) on LightCycler 480 (Roche Applied Science). Table 1 listed the primers and probes used in this study. Reactions were performed at $95{ }^{\circ} \mathrm{C}$ for 10 min followed by 45 cycles of $95{ }^{\circ} \mathrm{C}$ for $15 \mathrm{~s}$ and $60{ }^{\circ} \mathrm{C}$ for $1 \mathrm{~min}$. Expression levels were evaluated using the comparative threshold cycle methods.

\section{Immunocytochemistry}

OSCC cells were seeded on chamber slides and treated with zebularine and TSA. Cells were fixed with $4 \%$ paraformaldehyde and permeabilized using $0.2 \%$ Triton X-100 in PBS. After blocking, cells were incubated with anti-BEX4 antibodies (Abcam) and CF488-conjugated secondary antibodies (Biotium). Cells were visualized by fluorescent microscope (Nikon).

\section{Immunohistochemistry}

Tissue samples embedded into paraffin blocks were cut into 4- $\mu \mathrm{m}$ sections. After deparaffinization and rehydration, sections were microwaved in $10 \mathrm{mM}$ sodium citrate buffer for antigen retrieval and stained with anti-BEX4 antibodies (Abcam) at room temperature for $1 \mathrm{~h}$. DAKO EnVision + System, HRP (DAKO) was used for visualization of immunoreaction. Then, sections were stained with Mayer's hematoxylin, dehydrated and photographed under light microscope. BEX4 protein expression level was graded as low, moderate or high depending on the staining intensity. Association between BEX4 expression and the clinicopathological parameters of OSCC patients was evaluated by Chi-square test or Fisher's exact test.

\section{Lentivirus vector construction, lentivirus production and infection}

Full-length BEX4 coding sequence was cloned into lentiviral expression vector $\mathrm{pCDH}$ (System Biosciences) to generate the BEX4-expressing vector ( $\mathrm{pCDH}-\mathrm{BEX} 4$ vector). Vector sequences were verified by direct sequencing. Virus packaging was performed by transient transfection into 293 T cells using Lipofectamine 2000 Transfection Reagent (Invitrogen). OSCC cell lines CAL27 and YD-38 were transduced with medium containing lentivirus.

\section{SiRNA transfection}

BEX4 siRNA-1, BEX4 siRNA-2 and negative control siRNA (QIAGEN) were transfected into OSCC cells using HiPerFect transfection reagent (QIAGEN) according to the manufacturer's protocol. After $72 \mathrm{~h}$, cells were collected and the efficiency of BEX4 silencing was determined by qPCR and immunocytochemistry.

\section{Western blot}

His-tag BEX4 protein was obtained from Novus Biologicals. Protein was extracted from cells using RIPA lysis 
Table 1 Primers and probes for qPCR

\begin{tabular}{|c|c|c|c|}
\hline Name & Forward primer $\left(5^{\prime}-3^{\prime}\right)$ & Reverse primer $\left(5^{\prime}-3^{\prime}\right)$ & Probe number $^{a}$ \\
\hline BEX1 & CACCTCGTGGCGAGAATC & CTCTTTGGACTCCATTACTCCTG & 63 \\
\hline BEX2 & TCCTGAGGCTACGACCTTTC & CGATTCTCGACGTGAGGTG & 16 \\
\hline BEX3 & CTTCGGTGCAGTCGTCACT & ACACTTAGCCTCGCAGACCT & 24 \\
\hline BEX4 & GCCCCGAAATTAGGAAGC & TGTTTGCCGCTAGTTCCTCT & 15 \\
\hline BEX5 & GCCGATTTCAAGGCTAAGAG & GGACATTTCCATGTTGAGTTTT & 34 \\
\hline RSAD2 & CACGTAAACTAGATCAGGGAACAA & TCAACAAACTGCATGGGATATT & 55 \\
\hline S100A9 & CTCCCACGAGAAGATGCAC & GAGGCCTGGCTTATGGTG & 81 \\
\hline |F|6 & AACCGTTTACTCGCTGCTGT & GGGCTCCGTCACTAGACCTT & 40 \\
\hline PTPRZ1 & GGCCTGTTGTCGTCCACT & TCTGCTGCAACATACTGTCTAGC & 67 \\
\hline MS4A4A & GGAATTCTGCATTGCTGTGTC & TGATGGCAGAATTAACACAACC & 13 \\
\hline FABP5 & GAGTGGGATGGGAAGGAAAG & GATCCGAGTACAGGTGACATTG & 22 \\
\hline S100A7 & CCAAACACACACATCTCACTCA & TCAGCTTGAGTGTTGCTCATC & 33 \\
\hline S100A8 & GCCAAGCCTAACCGCTATAA & ATGATGCCCACGGACTTG & 34 \\
\hline SPRR1B & CAGAGTATTCCTCTCTTCACACCA & CAAGGCTGTTTCACCTGCT & 3 \\
\hline S100A12 & CACATTCCTGTGCATTGAGG & GGTGTCAAAATGCCCCTTC & 31 \\
\hline S100A7A & CATAGCCGCAGACTACCACA & ACTGGCTTCCCCCAGAAC & 21 \\
\hline SPRR1A & AGCCCAAGGTTCCAGAGC & TTCTGCTTGGTCTTCTGCTG & 67 \\
\hline |F|44 & AGCCTGTGAGGTCCAAGCTA & GAACATCCTTTACAGGGTCCAG & 6 \\
\hline PGLYRP3 & CCCCACTCCCTTTGAGACT & CTTGGCTGGTGAGGGTTG & 3 \\
\hline IL1R1 & GTCTCTGCTCTTGCCTCGAC & TCAGCTAGAGAAAATGCGAGAA & 63 \\
\hline CBX5 & AGAAGATGAAGGAGGGTGAAAA & GCGATATCATTGCTCTGCTCT & 7 \\
\hline PTX3 & TGTATGTGAATTTGGACAACGAA & CATTCCGAGTGCTCCTGAC & 58 \\
\hline PADI3 & CAGCAATGACCTCAACGACA & GAGGTAGAGCACCGCATAGG & 19 \\
\hline ANKRD22 & CAGTGCCTTTGTTTCATTGG & CACCTGTCAGTGCTITTCCA & 74 \\
\hline LIF & TGCCAATGCCCTCTITATTC & GTCCAGGTTGTTGGGGAAC & 26 \\
\hline TMEM156 & CATGGAATACCCGAATGATTG & CCAAGTGATCTTCATGGAACAA & 80 \\
\hline GPRC5B & CCGCAGAGATGTGACTCG & GATGCCACGAACATTCGAC & 78 \\
\hline FTH1 & GCCAGAACTACCACCAGGAC & CATCATCGCGGTCAAAGTAG & 1 \\
\hline SLC6A15 & AAAGTGGGGGCTTAAACTGG & CATAGCCAAGCAAACCATGA & 27 \\
\hline SLITRK6 & GAAGAAAAAGAAACTCAGGGATCA & GGACAGACTAAATTGCCTGAAGA & 7 \\
\hline PLLP & CACCCGGCCTTATAACCAG & AAGGCACTCACTCCATAGGC & 9 \\
\hline
\end{tabular}

aniversal ProbeLibrary

buffer. Protein concentration was measured by BCA protein assay system (Pierce). Proteins separated by sodium dodecyl sulphate-poly-acrylamide gel electrophoresis were transferred to PVDF membranes and incubated with antiBEX4 antibodies (Abcam). After incubation with horseradish peroxidase-conjugated goat anti-rabbit secondary antibody, hybridization signals were developed using ECL Plus Western Blotting Detection Reagents (Amersham Biosciences).

\section{Real-time proliferation assay}

Real-Time cell kinetic analyzer xCELLigence RTCA (ACEA Biosciences). was used to monitor the dynamic changes of cell proliferation. Data analysis was performed using RTCA Control Unit and the preinstalled RTCA software. For real-time proliferation assay, E-plate 16 was used. Cells were seeded directly onto E-plate. Changes in baseline impedance resulting from the increase of cell numbers were monitored by gold micro-electrodes located at the bottom of E-plate. The proportional changes in impedance were recorded continuously and expressed as cell index $(\mathrm{CI})$. Change of $\mathrm{CI}$ with time was monitored continuously for $72 \mathrm{~h}$.

\section{Cell cycle analysis}

Apoptosis was determined by propidium iodide (PI) staining. Cells were harvested and fixed with $70 \%$ ethanol for $24 \mathrm{~h}$ at $4{ }^{\circ} \mathrm{C}$. The cells were stained with PI and 
RNase A in PBS for 30 min in the dark. Cell-cycle distribution was analyzed with a FACS flow cytometer (BD Biosciences).

\section{Animal models}

Athymic nu/nu mice (5 weeks old, weight range: 18$22 \mathrm{~g})$ were used. CAL27 with pCDH-BEX4 vector or empty vector $\mathrm{pCDH}$ were injected subcutaneously into the right flank of mice. Four animals were used in each group. Tumor growth was measured daily with calipers in two dimensions. After 30 days, mice were euthanized. The animals were maintained under pathogen-free conditions with controlled temperature and humidity.

\section{Microarray}

Affymetrix whole-transcript expression microarray $\mathrm{Hu}-$ man Exon 1.0 ST was used. All the RNA samples were tested with Agilent 2100 Bioanalyser (Agilent Technologies). All the samples RNA had RIN value over 8 . All the tests were performed in the Centre for Genomic Sciences, the University of Hong Kong. Data analysis was evaluated using GeneSpring version 13 (Agilent Technologies). Genes with more than 1.5 -fold difference and $\mathrm{p}$ value below 0.01 were selected for $\mathrm{qPCR}$ validation.

\section{Statistical analysis}

Data were expressed as mean \pm standard deviation. $T$ test or Chi square test were used to compare difference between groups as appropriate. Data analysis was performed using SPSS $^{\circ}$ for Windows version 14.0 (SPSS, Inc., Chicago, IL, USA). All the tests were two-sided. Pvalues $<0.05$ were considered as statistically significant. SurvExpress (Interface v2.0, Database Update: Sep 30, 2015) was used to perform survival analysis on oral cancer dataset GSE26549 as described [12]. The dataset contains global gene expression data from 86 oral leukoplakia tissue samples examined with Affymetrix Human Gene 1.0 ST Array [13].

\section{Results}

\section{Aberrant expression of BEX4 in oral cancers}

To identify the key BEX family members involved in OSCC, we used qPCR to quantify BEX1-5 transcript levels in 65 primary OSCC tissues and compared with the paired normal epithelia. In comparison with the normal counterpart, expression of BEX1 $(p<0.001)$, BEX4 $(p<0.001)$ and $\operatorname{BEX} 5(p=0.006)$ were significantly reduced in the OSCC tissues (Fig. 1a). In contrast, BEX3 expression showed significant elevation in OSCC $(p=$ 0.026). For BEX2, no significant difference was observed between the OSCC and paired normal tissues $(p=0.457)$. The characteristics of the 65 patients examined were shown in Table 2.
Low BEX4 expression was associated with poor outcome in patients with oral pre-neoplastic lesions

We analyzed the association between BEX family member expression and the outcome of 86 oral leukoplakia patients from GSE26549 using SurvExpress. Low BEX4 expression was associated with poor cancer-free survival of oral leukoplakia patients (Hazard ratio $=2.76, p=$ 0.0072) (Fig. 1b, c). The correlation of low BEX4 expression with poor cancer-free survival in leukoplakia patients was more significant than other BEX family members including BEX1, BEX2, NGFRAP1 (BEX3) and BEX5 (Fig. 1c).

\section{Low BEX4 expression was associated with advanced tumor stage}

To confirm the specificity of BEX4 antibody, Western blot was performed on the his-tagged recombinant human BEX4 protein. Signal of the immunoreaction increased in parallel with the concentration of recombinant BEX4 (Fig. 2a). In tissues obtained from OSCC patients, majority of normal tissues (96.9 \%) showed moderate or high expression of BEX4. In the OSCC tissues, low expression of BEX4 was observed in $53.3 \%$ tumor samples from patients with OSCC, while it was only found in $3.3 \%$ paired normal tissues (Fig. 2b). Moreover, $75 \%$ tumor tissues with T3-4 stage exhibited low BEX4 expression. In contrast, only $10 \%$ tumor tissues with T1-2 stage displayed low BEX4 expression (Table 3). Low BEX4 level was associated with advanced stage tumor $(p=0.001$, Table 3$)$. Representative images of BEX4 staining on oral tissues were shown in Fig. 2c.

\section{BEX4 was epigenetically silenced in OSCC}

Next, we sought to examine whether DNA methylation and histone acetylation are the major epigenetic alterations leading to BEX4 suppression in OSCC. DNA methylation was controlled by DNA methyltransferases that catalyze the addition of a methyl group to DNA. Zebularine inhibited DNA methyltransferases through the formation of a covalent complex with them [14]. Histone deacetylases (HDACs) catalyze the removal of acetyl groups of histone, leading to chromatin condensation and transcriptional suppression. TSA was a specific inhibitor of HDAC activity [15]. Following treatment with zebularine and TSA, dose-depending increase in BEX4 expression was observed in CAL27 cells (Fig. 3). The protein expression level of BEX4 was also enhanced following zebularine and TSA treatment (Fig. 3).

BEX4 reduced the proliferation and tumor growth of OSCC Having established that BEX4 expression was suppressed in OSCC, we hypothesized that BEX4 has a tumor suppressing function in OSCC. We generated BEX4overexpressing OSCC cell lines (Fig. $4 \mathrm{a}-\mathrm{c}$ ) and measured 


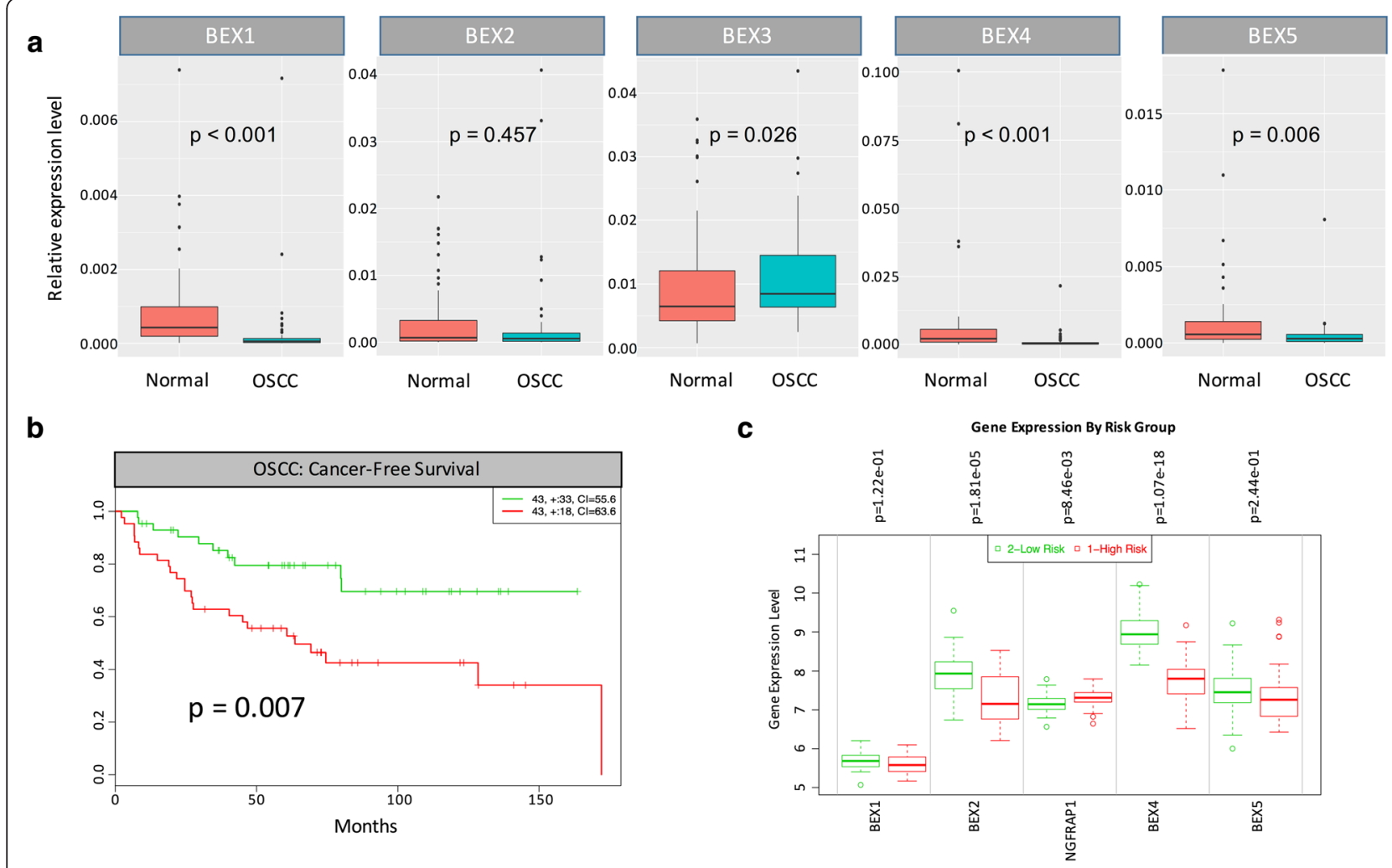

Fig. 1 Expression of BEX family members in OSCC. a Boxplots showing expression level of BEX family members in OSCC and paired normal tissues. Each sample were normalized to GAPDH and displayed using $2^{-\Delta C t}$ values. $\mathbf{b}$ Cox survival analysis of cancer-free survival was performed on oral leukoplakia patients based on the BEX4 expression expression level. c Boxplots showing the expression level of BEX family members in different risk group

Table 2 Association of BEX4 mRNA expression level with the clinicopathological variables in 65 patients with OSCC

\begin{tabular}{|c|c|c|c|c|}
\hline \multirow[t]{2}{*}{ Variables } & \multirow[t]{2}{*}{ Cases } & \multicolumn{2}{|c|}{ BEX4 expression } & \multirow[t]{2}{*}{$P$ value* } \\
\hline & & Low (\%) & High (\%) & \\
\hline Gender & - & - & - & 0.875 \\
\hline Male & 40 & $20(50)$ & $20(50)$ & - \\
\hline Female & 25 & $13(52)$ & $12(48)$ & - \\
\hline Age & - & - & - & 0.708 \\
\hline$>59$ & 32 & $17(53)$ & $15(47)$ & - \\
\hline$\leq 59$ & 33 & $16(48)$ & $17(52)$ & - \\
\hline T-stage & - & - & - & 0.174 \\
\hline $\mathrm{T} 1-2$ & 29 & $12(41)$ & $17(59)$ & - \\
\hline T3-4 & 36 & $21(58)$ & $15(42)$ & - \\
\hline Nodal stage & - & - & - & 0.694 \\
\hline Negative & 37 & $18(49)$ & $19(51)$ & - \\
\hline Positive & 28 & $15(54)$ & $13(46)$ & - \\
\hline
\end{tabular}

*Pearson chi-square test the change of proliferation rate. BEX4-overexpressing cells showed significant reduction in OSCC proliferation (Fig. 4d, e). Apoptotic cells were determined by PI staining using flow cytometry. Flow cytometry analysis showed that BEX4 overexpression in OSCC did not induce apoptosis of OSCC (Fig. 4f). To confirm our results, two different BEX4-specific siRNAs were used to silence the expression of BEX4 in OSCC cells (Fig. 5a, b). Silence of BEX4 resulted in a significant increase in the proliferation of CAL27 and YD-38 cells (Fig. 5c, d).

In the animal model, BEX4-overexpressing xenograft developed from CAL27 cells had a significantly smaller tumor volume and slower growth rate (Fig. 6). Tumor volume of control xenograft increased by 4.7 -fold from day 17 to day 53. In contrast, tumor volume of BEX4overexpressing xenograft increased by only 1.7 -fold (Fig. 6). The data is consistent with the cell line models suggesting that BEX4 was involved in controlling OSCC growth.

\section{S100A family is the potential downstream targets regulated by BEX4}

As there is no sufficient information about the regulatory mechanisms of BEX4 in human diseases, we used 


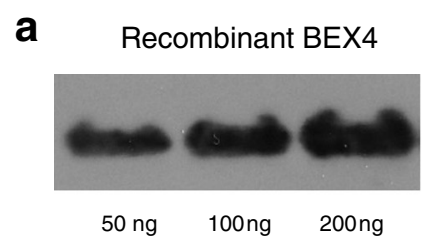

\section{C

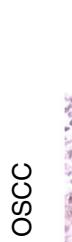

b
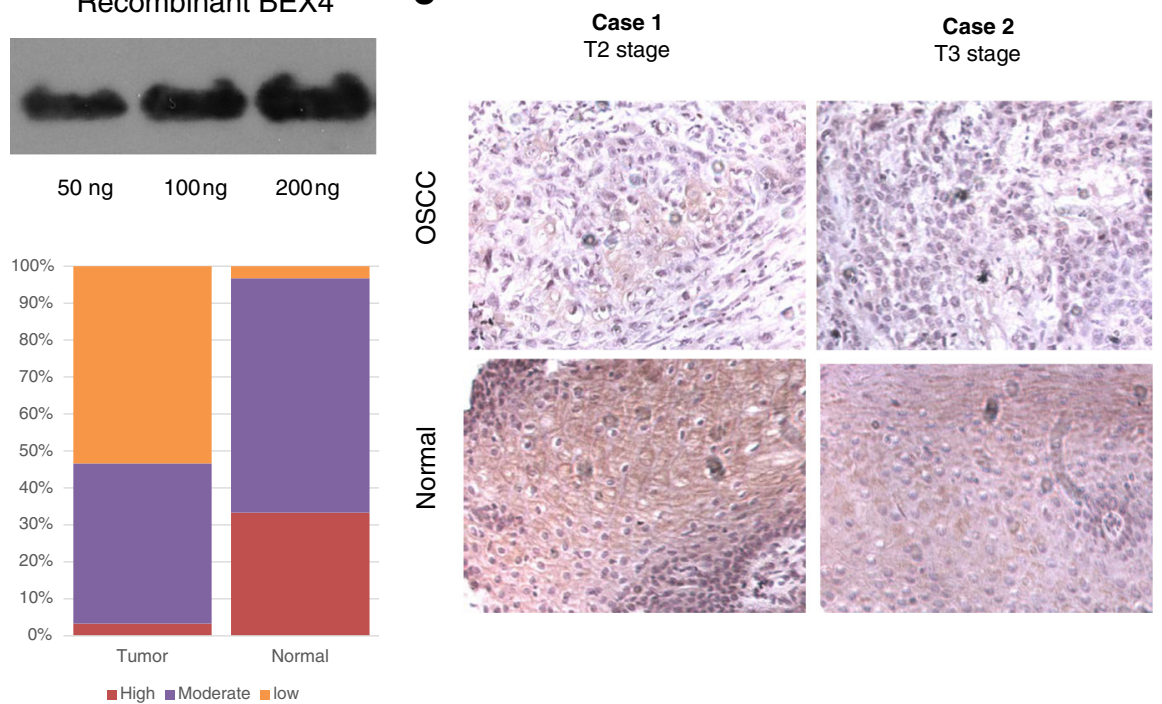

- High $=$ Moderate $\backsim$ low

Fig. 2 Immunohistochemical analysis of BEX4 expression in OSCC. In total, 30 paired tumor and normal tissues from patients with OSCC. a Specificity of BEX4 antibody. Western bot analysis showed that the anti-human BEX4 antibody can specifically detect the recombinant human BEX4 protein. b Distribution of tissue BEX4 expression level in our OSCC cohort. $\mathbf{c}$ Representative immunohistochemical staining images showing BEX4 expression on OSCC tissues and paired normal epithelia

microarray to explore the global gene expression changes in BEX4-overexpressing OSCC cell line CAL27. Twentysix genes showed differential expression (Table 4). The filtered genes were then validated in BEX4-overexpressing Cal27 and YD-38 again. Of which, the expression patterns of 16 genes were consistent with the microarray results (Fig. 7). According to the expression patterns in $\mathrm{qPCR}$

Table 3 Association of BEX4 protein expression level with the clinicopathological variables in 30 patients with OSCC

\begin{tabular}{cllll}
\hline Variables & Cases & \multicolumn{2}{l}{ BEX4 expression } & P value \\
\cline { 3 - 4 } & & Low (\%) & Moderate +High (\%) & \\
\hline Gender & - & - & - & $0.796^{\mathbf{a}}$ \\
Male & 20 & $11(55)$ & $9(45)$ & - \\
Female & 10 & $5(50)$ & $5(50)$ & - \\
Age & - & - & - & $0.464^{\mathrm{a}}$ \\
$\leq 54$ & 15 & $9(60)$ & $6(40)$ & - \\
$>54$ & 15 & $7(47)$ & $8(53)$ & - \\
T-stage & - & - & - & $\mathbf{0 . 0 0 1 ^ { \mathbf { b } }}$ \\
T1-2 & 10 & $1(10)$ & $9(90)$ & - \\
T3-4 & 20 & $15(75)$ & $5(25)$ & - \\
Nodal stage & - & - & - & $0.464^{\mathbf{a}}$ \\
Negative & 15 & $7(47)$ & $8(53)$ & - \\
Positive & 15 & $9(60)$ & $6(40)$ & - \\
\hline
\end{tabular}

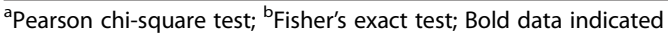
statistically significant results, we classified the genes into BEX4-suppressed genes (S100A7, S100A8, S100A7A, RSAD2, S100A12, SPRR1B, S100A9, SPRR1A, MS4A4A, IF16, PGLYRP3, IL1R1, IFI44, FABP5, PTPRZ1) and BEX4-induced genes (FTH1, PLLP, SLC6A15, PTX3, CBX5, TMEM156, GPRC5B, ANKRD22, LIF, PADI3, SLITRK6). Among the differentially expressed genes, expression changes of multiple S100A family members were observed in BEX4overexpressing Cal27 and YD-38. S100A7, S100A7A, S100A8, S100A9 and S100A12 were remarkably increased in the BEX4-overexpressing OSCC cell lines.

\section{Discussion}

Our results indicated that BEX4 functions as tumor suppressor in controlling proliferation and growth of OSCC. At present, little is known about the expression status and functions of brain expressed X-linked family in OSCC. Thus, we first examined the mRNA levels of BEX1, BEX2, BEX3, BEX4 and BEX5 in our OSCC tissues and compared with the paired normal epithelia. We identified that BEX4 suppression is a common event in our OSCC and is potentially associated with the poor outcome of patients. The association between low BEX4 with poor outcome of patients with oral pre-neoplastic lesions suggested that reduced BEX4 is an early event in the development of OSCC.

OSCC is a male dominant disease. Recent epidemiology study revealed that there is an increasing number of female OSCC cases [16]. In our cohort, reduced 

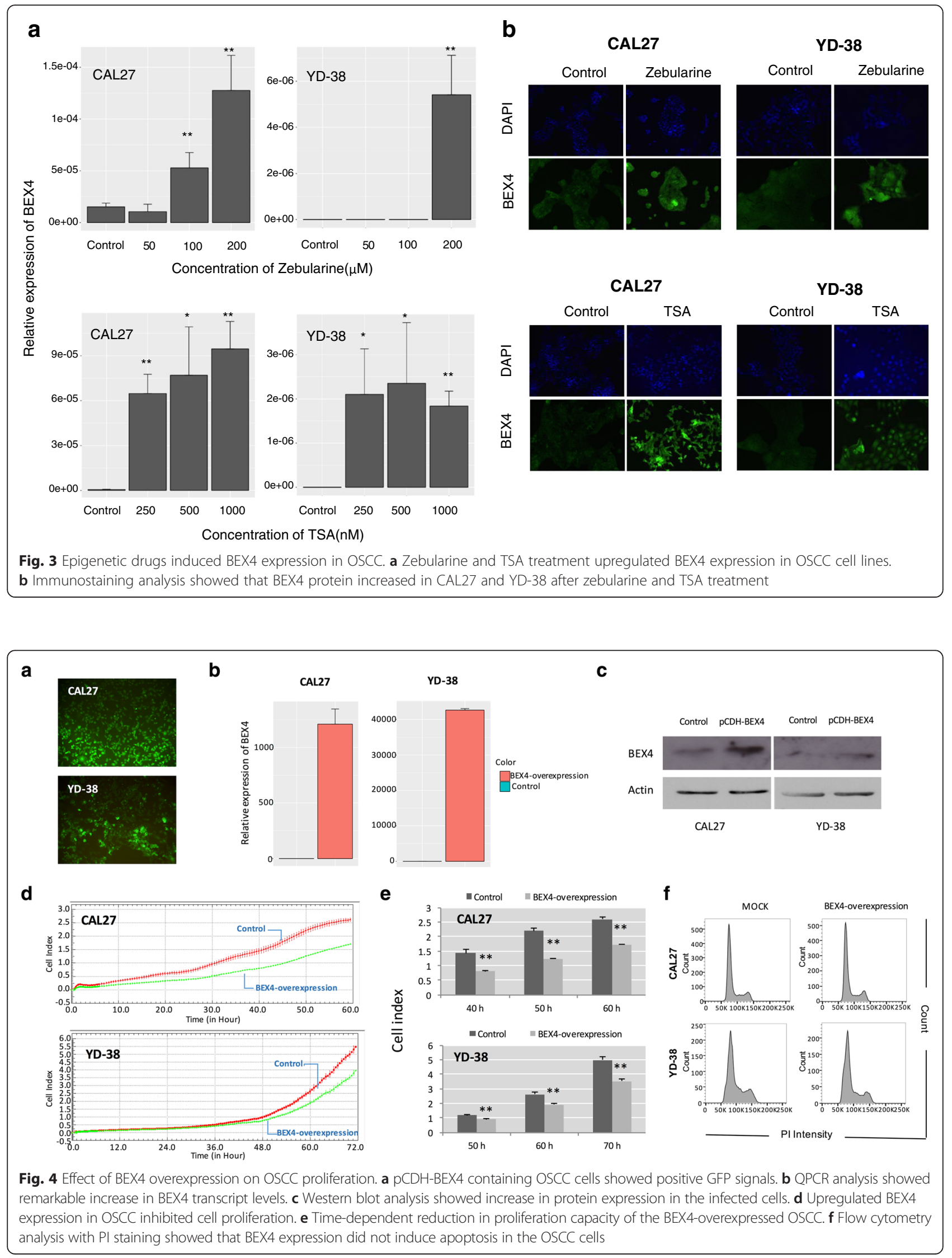


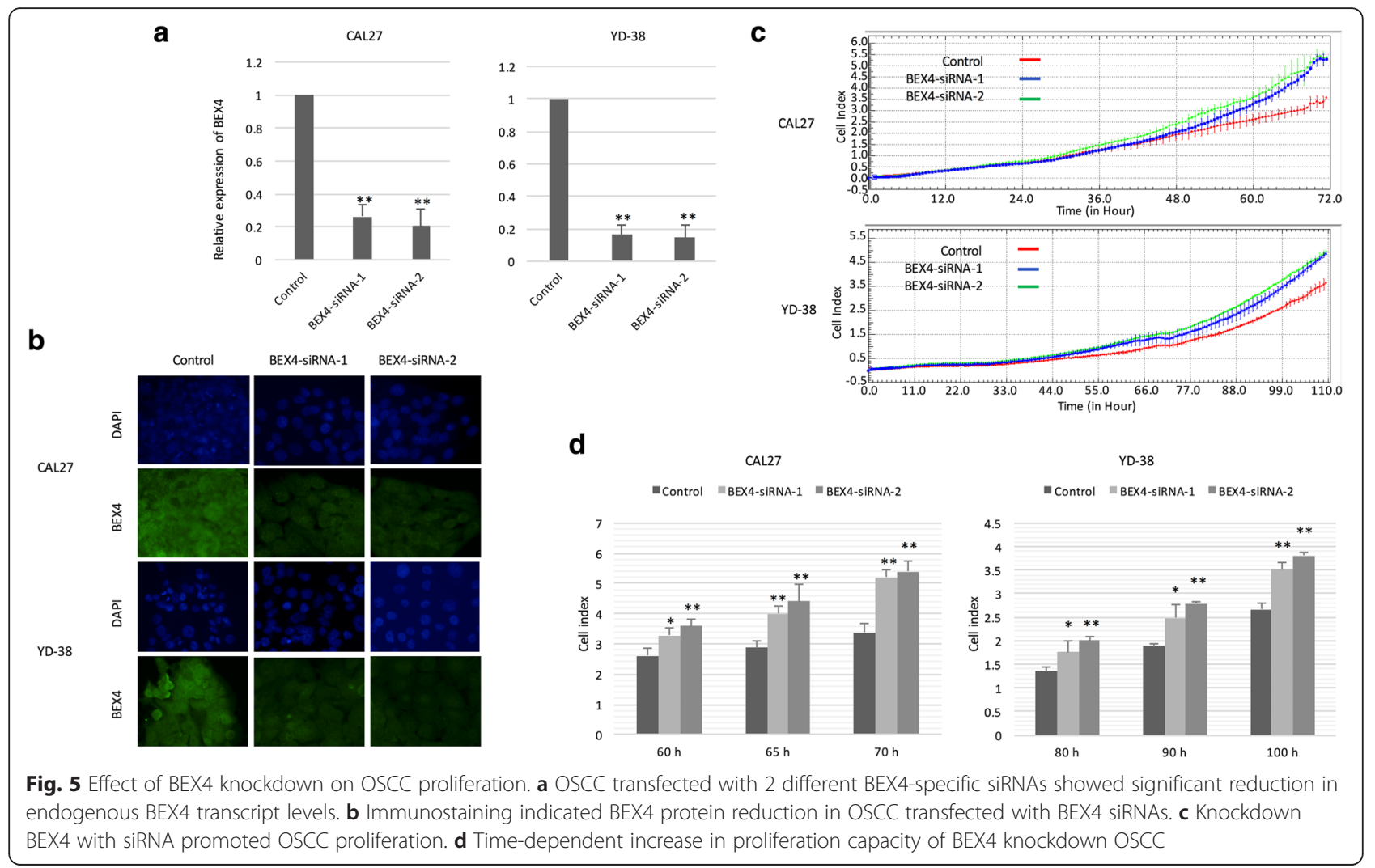

BEX4 expression is also observed in the female group. Functional analysis revealed that BEX4 reduced proliferation propensity of OSCC. The remarkable reduction of xenograft volume further supports our suggestion that BEX4 controls the proliferative growth process of OSCC. Taken together, our results provide evidence showing a putative tumor-suppressing functions of BEX4 in OSCC.

Members of BEX family have been implicated in regulating apoptosis in various human cancer cells and normal cells. In breast cancer, BEX2 could modulate ceramide-induced apoptosis via protein phosphatase $2 \mathrm{~A}$ $[17,18]$. BEX1 interacted with B-cell lymphoma 2 (BCL2) and inhibited the formation of BCL-2/BCL-2-associated $\mathrm{X}$ protein (BAX) complex, leading to enhancement of imatinib-induced apoptosis in human leukemic cell line K562 [19]. BEX3 could interact with the death domain of p75 neurotrophin receptor (p75NTR) and induce apoptosis upon nerve growth factor (NGF) treatment in HEK293 cells [20]. In light of the regulating role of BEX family in apoptosis, we investigated whether BEX4

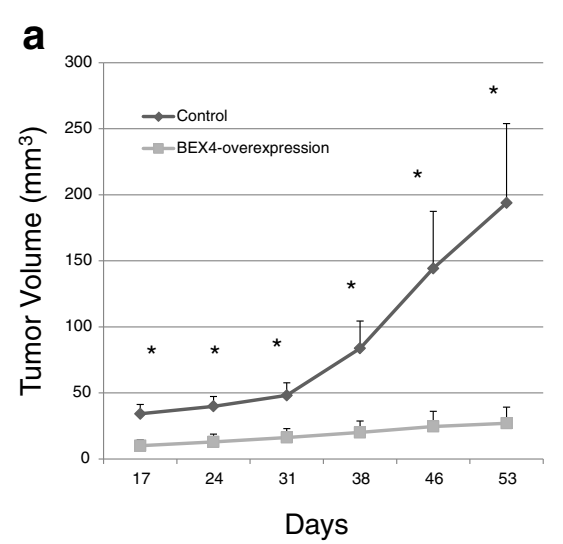

b
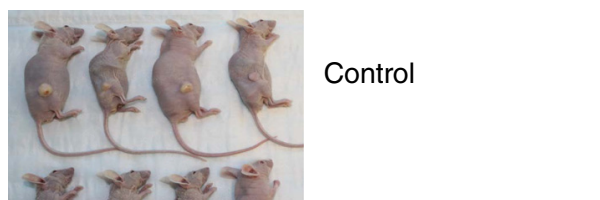

BEX4-overexpression

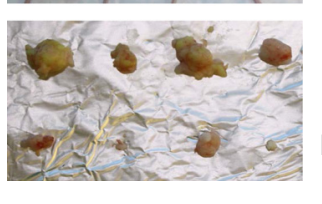

Control

BEX4-overexpression

Fig. 6 BEX4 inhibited growth of CAL27 xenograft. a Xenograft growth curve. $\mathbf{b}$ Xenograft excised from the indicated time point 
Table 4 Gene expression profile of BEX4-overexpressing CAL27

\begin{tabular}{|c|c|c|c|c|}
\hline Transcripts cluster Id & Gene symbol & Regulation & Fold-change & Gene name \\
\hline 2436051 & S100A7 & Up & 3.03 & S100 Calcium Binding Protein A7 \\
\hline 2435989 & S100A8 & Up & 2.80 & S100 Calcium Binding Protein A8 \\
\hline 2359691 & S100A7A & Up & 2.47 & S100 Calcium Binding Protein A7A \\
\hline 2468351 & RSAD2 & Up & 2.00 & Radical S-Adenosyl Methionine Domain Containing 2 \\
\hline 2435981 & S100A12 & Up & 1.79 & S100 Calcium Binding Protein A12 \\
\hline 2359521 & SPRR1B & Up & 1.79 & Small Proline-Rich Protein 1B \\
\hline 2359664 & S100A9 & Up & 1.72 & S100 Calcium Binding Protein A9 \\
\hline 2359492 & SPRR1A & Up & 1.68 & Small Proline-Rich Protein 1A \\
\hline 3332298 & MS4A4A & Up & 1.65 & Membrane-Spanning 4-Domains, Subfamily A, Member 4A \\
\hline 2403261 & IFI6 & Up & 1.64 & Interferon, Alpha-Inducible Protein 6 \\
\hline 2435949 & PGLYRP3 & Up & 1.59 & Peptidoglycan Recognition Protein 3 \\
\hline 2496962 & IL1R1 & Up & 1.58 & Interleukin 1 Receptor, Type I \\
\hline 2343511 & IF|44 & Up & 1.56 & Interferon-Induced Protein 44 \\
\hline 3104933 & FABP5 & Up & 1.54 & Fatty Acid Binding Protein 5 (Psoriasis-Associated) \\
\hline 3021377 & PTPRZ1 & Up & 1.52 & Protein Tyrosine Phosphatase, Receptor-Type, Z Polypeptide 1 \\
\hline 4037708 & FTH1 & Down & 2.39 & Ferritin, Heavy Polypeptide 1 \\
\hline 3693141 & PLLP & Down & 1.67 & Plasmolipin \\
\hline 3464276 & SLC6A15 & Down & 1.61 & Solute Carrier Family 6 (Neutral Amino Acid Transporter), Member 15 \\
\hline 2649367 & PTX3 & Down & 1.60 & Pentraxin 3, Long \\
\hline 3456630 & CBX5 & Down & 1.59 & Chromobox Homolog 5 \\
\hline 2766289 & TMEM156 & Down & 1.54 & Transmembrane Protein 156 \\
\hline 3683377 & GPRC5B & Down & 1.54 & G Protein-Coupled Receptor, Class C, Group 5, Member B \\
\hline 3299469 & ANKRD22 & Down & 1.54 & Ankyrin Repeat Domain 22 \\
\hline 3957160 & LIF & Down & 1.54 & Leukemia Inhibitory Factor \\
\hline 2322818 & PADI3 & Down & 1.52 & Peptidyl Arginine Deiminase, Type III \\
\hline 3519840 & SLITRK6 & Down & 1.51 & SLIT And NTRK-Like Family, Member 6 \\
\hline
\end{tabular}

overexpression affect apoptosis in OSCC. Unlike other BEX family proteins, BEX4 had no inducing effect on apoptosis and cell cycle arrest.

At present, the functional roles of BEX family members in cancer remain unclear. In OSCC, it has been reported that $\mathrm{BEX} 1$ is involved in modulating nuclear factor- $\kappa B$ (NF- $\kappa B)$ signaling pathway [8]. BEX2 expression could possibly be modulated by the aberrantly activated mechanistic target of rapamycin (mTOR) in cancer cells [21]. BEX protein family is also known as intrinsically disordered proteins (IDPs), which exist as dynamic ensemble of conformation with no discrete 3D structure. IDPs are suggested to play an important role in tumorigenesis and are involved in modulating multiple gene regulating signaling pathways by their ability to recognize multiple interaction partners [22]. Our results revealed that BEX family member, $\mathrm{BEX} 4$, is one of the IDP involved in the pathogenesis of OSCC.

Epigenetic silencing of tumor suppressor gene is a characteristic feature of OSCC. Existing data suggests that DNA methylation and chromatin remodeling play an important role in modulating BEX family expression in human malignancies [23, 24]. In glioma, BEX1 and $\mathrm{BEX} 2$ are silenced and the expression could be activated by treatment with DNA methyltransferase inhibitor and histone deacetylase inhibitor [25]. Our results are consistent with the current knowledge suggesting that BEX4 is epigenetically silenced in OSCC.

OSCC overexpressing BEX4 showed significant upregulation of S100A family members (S100A7, S100A8, S100A9, S100A12, S100A15 or S100A7A). S100A is a family of calcium binding protein involved in multiple processes of cancer development [26]. Of which, S100A7 dysregulation is believed to be involved in a wide range of pathological processes associated with OSCC. S100A7 (also known as psoriasin) was expressed in early stage oral cancer. S100A7 expression was reduced in tumor in late stage [27]. Additionally, highly differentiated OSCC usually had high expression of S100A7 as compared to the moderate or poorly differentiated counterparts [28]. 


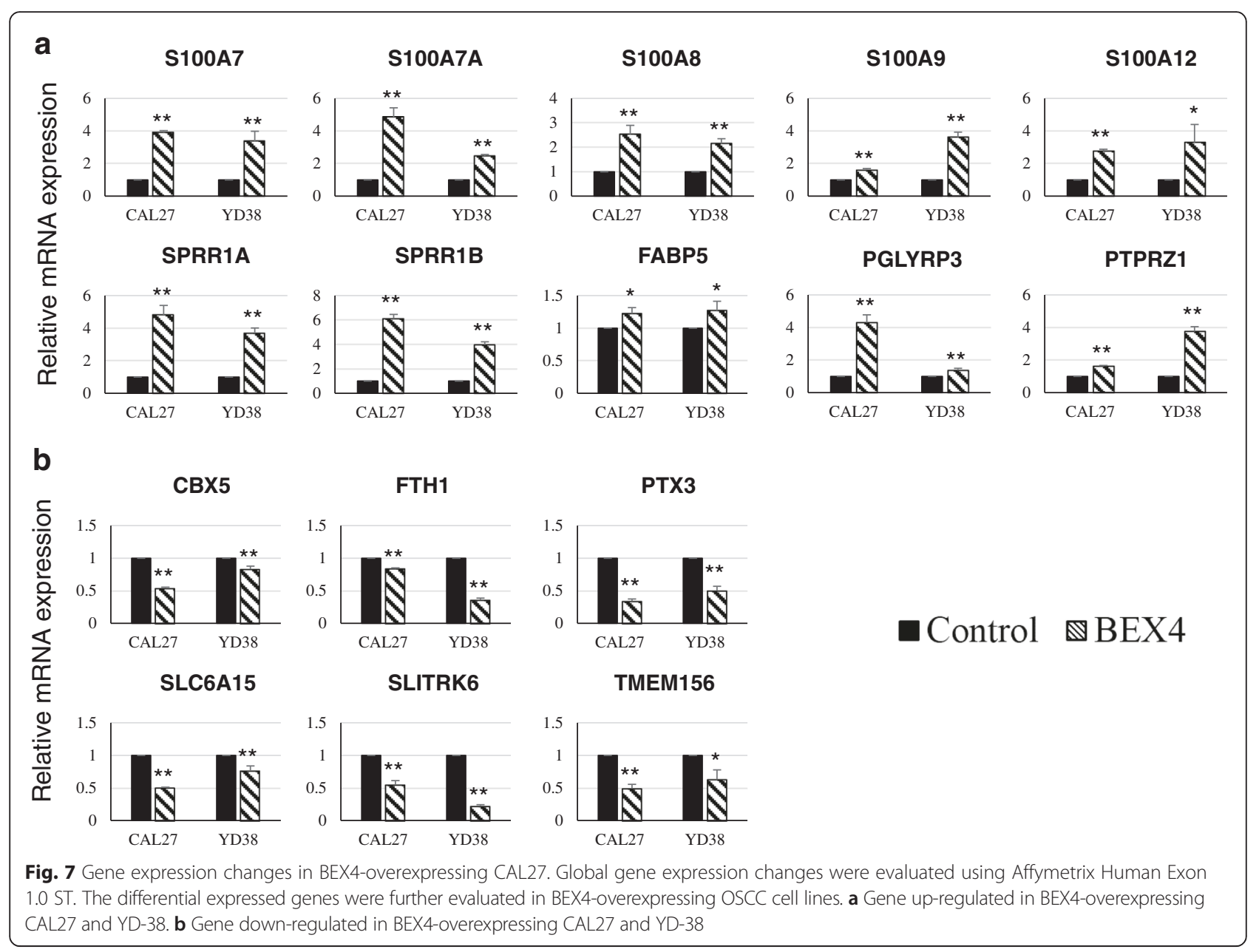

Forced expression of S100A7 is accompanied by repressed cell proliferation and tumor progression in orthotopic tongue tumor model [27].

Apart from S100A7, aberrant expression of other S100A family members is emerging as an important event in head and neck cancers. In head and neck squamous cell carcinoma, S100A8/A9 (calprotectin) could suppress cell growth by inducing G2/M cell cycle checkpoint arrest [29]. The mRNA and protein expression levels of S100A8/ A9 are decreased in head and neck cancers. S100A8/A9 can modulate G2/M Cdc2/cyclin B1 complex activation resulting in G2/M checkpoint arrest [29]. For S100A12, high level in tumor tissue is a good prognostic indicator for oropharyngeal squamous cell carcinoma patient [30]. S100A7A (koebnerisin) are highly homologous to S100A7. Both S100A7A and S100A7 could serve as oncogenic inactivator in human malignancies [31].

The molecular mechanisms underlying S100A proteins dysregulation remains unclear. In OSCC, it has been shown that overexpression of $\beta$-catenin decreased the expression of S100A7, while silence of $\beta$-catenin enhanced its expression, indicating that $\beta$-catenin signaling negatively modulated S100A7 [27]. In addition, c-Myc suppressed S100A7 expression by directly binding to its promoter [32].

\section{Conclusions}

Based on the current results, we suggested that BEX4 controls OSCC proliferation. Although the mechanisms underlying BEX4 suppression in OSCC remains unresolved, induced expression of BEX4 in the presence of epigenetic drugs suggested that BEX4 is epigenetically silenced in OSCC. Taken together, we suggested that BEX4 functions as a novel tumor suppressor gene involved in OSCC.

\section{Abbreviations}

BAX: BCL-2-associated X protein; BCL-2: B-cell lymphoma 2; BEX: brainexpressed X-linked; HDACs: histone deacetylases; HNSCC: head and neck squamous cell carcinoma; mTOR: mechanistic target of rapamycin; NGF: nerve growth factor; OSCC: oral squamous cell carcinoma; p75NTR: p75 neurotrophin receptor; TSA: trichostatin A.

\section{Acknowledgement}

The study was support by Seed Funding for Basic Research, the University of Hong Kong. 


\section{Authors' contributions}

WG performed the experimental studies and prepared the manuscript. ZHL, SQC and CYC generated the vectors and conducted the animal experiments. JWYC participated in the study design and clinical sample collection. TSW design the project and is involved in data analysis and manuscript preparation. All authors read and approved the final manuscript.

\section{Competing interests}

The authors declare that they have no competing interests.

\section{Author details}

'Department of Surgery, The University of Hong Kong, Hong Kong SAR, China. 'Department of Otolaryngology, The First People's Hospital of Foshan, Foshan, Guangdong Province, China.

\section{Received: 1 October 2015 Accepted: 3 May 2016 \\ Published online: 13 June 2016}

\section{References}

1. Warnakulasuriya S. Global epidemiology of oral and oropharyngeal cancer. Oral Oncol. 2009:45:309-16.

2. Warnakulasuriya S. Living with oral cancer: epidemiology with particular reference to prevalence and life-style changes that influence survival. Oral Oncol. 2010;46:407-10.

3. Li J, Yang D, Wang W, Piao S, Zhou J, Saiyin W, et al. Inhibition of autophagy by 3-MA enhances IL-24-induced apoptosis in human ora squamous cell carcinoma cells. J Exp Clin Cancer Res. 2015;34:97.

4. Jerjes W, Upile T, Petrie A, Riskalla A, Hamdoon Z, Vourvachis M, et al. Clinicopathological parameters, recurrence, locoregional and distant metastasis in 115 T1-T2 oral squamous cell carcinoma patients. Head Neck Oncol. 2010;2:9.

5. Noguchi M, Kinjyo H, Kohama G-I, Nakamori K. Invasive front in oral squamous cell carcinoma: image and flow cytometric analysis with clinicopathologic correlation. Oral Surg Oral Med Oral Pathol Oral Radiol Endod. 2002:93:682-7.

6. Bonner JA, Harari PM, Giralt J, Cohen RB, Jones CU, Sur RK, et al. Radiotherapy plus cetuximab for locoregionally advanced head and neck cancer: 5-year survival data from a phase 3 randomised trial, and relation between cetuximab-induced rash and survival. Lancet Oncol. 2010;11:21-8.

7. Chen LH, Liu DW, Chang JL, Chen PR, Hsu LP, Lin HY, et al. Methylation status of insulin-like growth factor-binding protein 7 concurs with the malignance of oral tongue cancer. J Exp Clin Cancer Res. 2015;34:20.

8. Lee CH, Wong TS, Chan JYW, Lu SC, Lin P, Cheng AJ, et al. Epigenetic regulation of the $X$-linked tumour suppressors BEX1 and LDOC 1 in oral squamous cell carcinoma. J Pathol. 2013;230:298-309.

9. Zhang L. Adaptive evolution and frequent gene conversion in the brain expressed X-linked gene family in mammals. Biochem Genet. 2008;46:293-311.

10. Vilar M, Murillo-Carretero M, Mira H, Magnusson K, Besset V, Ibáñez CF. Bex1, a novel interactor of the $\mathrm{p} 75$ neurotrophin receptor, links neurotrophin signaling to the cell cycle. EMBO J. 2006:25:1219-30.

11. Zhou X, Meng Q, Xu X, Zhi T, Shi Q, Wang Y, et al. Bex2 regulates cell proliferation and apoptosis in malignant glioma cells via the c-Jun $\mathrm{NH}_{2}$ terminal kinase pathway. Biochem Biophys Res Commun. 2012;427:574-80.

12. Saintigny $P$, Zhang L, Fan Y-H, El-Naggar AK, Papadimitrakopoulou VA, Feng $L$, et al. Gene expression profiling predicts the development of oral cancer. Cancer Prev Res (Phila). 2011;4:218-29.

13. Aguirre-Gamboa R, Gomez-Rueda H, Martínez-Ledesma E, Martínez-Torteya A, Chacolla-Huaringa R, Rodriguez-Barrientos A, et al. SurvExpress: an online biomarker validation tool and database for cancer gene expression data using survival analysis. PLoS One. 2013;8:e74250.

14. Zhou L, Cheng X, Connolly BA, Dickman MJ, Hurd PJ, Hornby DP. Zebularine: a novel DNA methylation inhibitor that forms a covalent complex with DNA methyltransferases. J Mol Biol. 2002;321:591-9.

15. Liu X, Chen X, Yu X, Tao Y, Bode AM, Dong Z, et al. Regulation of microRNAs by epigenetics and their interplay involved in cancer. J Exp Clin Cancer Res. 2013:32:96

16. Farshadpour F, Hordijk GJ, Koole R, Slootweg PJ. Non-smoking and nondrinking patients with head and neck squamous cell carcinoma: a distinct population. Oral Dis. 2007;13:239-43.

17. Naderi A, Teschendorff AE, Beigel J, Cariati M, Ellis IO, Brenton JD, et al. BEX2 is overexpressed in a subset of primary breast cancers and mediates nerve growth factor/nuclear factor-kappaB inhibition of apoptosis in breast cancer cell lines. Cancer Res. 2007:67:6725-36.

18. Naderi A, Liu J, Bennett IC. BEX2 regulates mitochondrial apoptosis and G1 cell cycle in breast cancer. Int J Cancer. 2010;126:1596-610.

19. Xiao Q, Hu Y, Liu Y, Wang Z, Geng H, Hu L, et al. BEX1 promotes imatinibinduced apoptosis by binding to and antagonizing BCL-2. PLoS One. 2014;9:e91782

20. Mukai J, Shoji S, Kimura MT, Okubo S, Sano H, Suvanto P, et al. Structurefunction analysis of NADE: identification of regions that mediate nerve growth factor-induced apoptosis. J Biol Chem. 2002;277:13973-82.

21. Hu Z, Wang Y, Huang F, Chen R, Li C, Wang F, et al. Brain expressed $X$-linked 2 is pivotal for hyperactive mTOR-mediated tumorigenesis. J Biol Chem. 2015:290(42):25756-65.

22. Fernandez EM, Díaz-Ceso MD, Vilar M. Brain expressed and X-linked (Bex) proteins are intrinsically disordered proteins (IDPs) and form new signaling hubs. PLoS One. 2015;10:e0117206.

23. Fischer C, Drexler HG, Reinhardt J, Zaborski M, Quentmeier H. Epigenetic regulation of brain expressed $X$-linked-2, a marker for acute myeloid leukemia with mixed lineage leukemia rearrangements. Leukemia. 2007;21:374-7.

24. Röhrs S, Dirks WG, Meyer C, Marschalek R, Scherr M, Slany R, et al. Hypomethylation and expression of BEX2, IGSF4 and TIMP3 indicative of MLL translocations in acute myeloid leukemia. Mol Cancer. 2009:8:86.

25. Foltz G, Ryu GY, Yoon JG, Nelson T, Fahey J, Frakes A, et al. Genome-wide analysis of epigenetic silencing identifies BEX1 and BEX2 as candidate tumor suppressor genes in malignant glioma. Cancer Res. 2006;66:6665-74.

26. Chen $\mathrm{H}, \mathrm{Xu}$ C, Jin O, Liu Z, S100 protein family in human cancer. Am J Cancer Res. 2014:4:89-115.

27. Zhou G, Xie TX, Zhao M, Jasser SA, Younes MN, Sano D, et al. Reciprocal negative regulation between $\mathrm{S100A7/psoriasin}$ and beta-catenin signaling plays an important role in tumor progression of squamous cell carcinoma of oral cavity. Oncogene. 2008;27:3527-38.

28. Kesting MR, Sudhoff $H$, Hasler RJ, Nieberler M, Pautke C, Wolff K-D, et al. Psoriasin (S100A7) up-regulation in oral squamous cell carcinoma and its relation to clinicopathologic features. Oral Oncol. 2009;45:731-6.

29. Khammanivong A, Wang C, Sorenson BS, Ross KF, Herzberg MC. S100A8/A9 (calprotectin) negatively regulates $\mathrm{G} 2 / \mathrm{M}$ cell cycle progression and growth of squamous cell carcinoma. PLoS One. 2013:8:e69395.

30. Funk S, Mark R, Bayo P, Flechtenmacher C, Grabe N, Angel P, et al. High S100A8 and $\mathrm{S100A} 12$ protein expression is a favorable prognostic factor for survival of oropharyngeal squamous cell carcinoma. Int J Cancer. 2015;136:2037-46.

31. Hattinger E, Zwicker S, Ruzicka T, Yuspa SH, Wolf R. Opposing functions of psoriasin (S100A7) and koebnerisin (S100A15) in epithelial carcinogenesis. Curr Opin Pharmacol. 2013;13:588-94

32. Kennedy RD, Gorski JJ, Quinn JE, Stewart GE, James CR, Moore S, et al. BRCA1 and c-Myc associate to transcriptionally repress psoriasin, a DNA damage-inducible gene. Cancer Res. 2005;65:10265-72.

\section{Submit your next manuscript to BioMed Central and we will help you at every step:}

- We accept pre-submission inquiries

- Our selector tool helps you to find the most relevant journal

- We provide round the clock customer support

- Convenient online submission

- Thorough peer review

- Inclusion in PubMed and all major indexing services

- Maximum visibility for your research

Submit your manuscript at www.biomedcentral.com/submit 